Check for updates

Cite this: RSC Adv., 2018, 8, 13266

Received 13th September 2017

Accepted 27th March 2018

DOI: 10.1039/c7ra10172d

rsc.li/rsc-advances

\section{Effect of dietary chitosan oligosaccharide supplementation on the pig ovary transcriptome}

\author{
Qingsong Xu, (D) *a Chen Qu, ${ }^{a}$ Jin Wan, (D) ${ }^{b}$ Gong Cheng, ${ }^{\text {cd }}$ Wen Yang, ${ }^{a}$ \\ Changhao Gong, ${ }^{a}$ Jun $\mathrm{He}\left(\mathbb{D}{ }^{* b}\right.$ and Yuguang $\mathrm{Du}^{\mathrm{c}}$
}

Fecundity improvement is one of the most important economic traits for the swine industry as it significantly increases production efficiency. Intriguingly, chitosan oligosaccharide (COS), a biomaterial with an active amino group, could promote sow reproductive performance. Therefore, we investigated the effects of dietary COS supplementation on the gene expression differences in the ovaries of sows using the RNASeq method. This analysis obtained 13960051 and 14564863 clean reads in control ovary and COS ovary libraries, respectively. A total of 486 differentially expressed genes (DEGs) were thereby identified (FDR $\leq 0.001, \mid \log _{2}$ ratio $\geq 1$ ). There were 234 up-regulated and 252 down-regulated genes in the COS ovary samples compared with the control ovary samples. A large number of these DEGs were involved in the terms cellular process, cell \& cell part and binding. Furthermore, pathway analysis indicated that these DEGs were significantly enriched in 34 Kyoto Encyclopedia of Genes and Genomes (KEGG) pathways, including cell cycle, progesterone-mediated oocyte maturation, metabolic pathways, oocyte meiosis, and hematopoietic cell lineage among others. These results provided the molecular mechanisms of using COS feed additive for improving sow litter size and prolificacy.

\section{Introduction}

In the swine production industry, litter size is one of the most meaningful economic traits and varies among individual animals. However, reproductive traits in swine are complex, from ovulation, fertilization, and implantation through to the birth of piglets, every process may affect litter size. ${ }^{1}$ Recently, various efforts have been made to investigate factors influencing litter size, including genetic factors, management of sows, optimizing nutrition and husbandry. ${ }^{2}$ It is well known that sow diet and health during gestation are important for foetal survival and sow reproductive performance. ${ }^{3}$ Nutrition optimization for increased litter size has in turn improved the prolificacy of sows over the past ten years. For instance, some functional oligosaccharides ${ }^{4}$ and amino acid $^{5}$ have been used to improve the pregnant animal reproductive performance. Nonetheless, the molecular basis of nutrition improvement and sow prolificacy remains largely unknown.

With the rapid development of sequencing technique and bioinformatics analysis, RNA-Seq technology provides a platform for measuring large-scale gene expression pattern. ${ }^{6}$ It has

\footnotetext{
${ }^{a}$ Liaoning Key Laboratory of Marine Animal Immunology and Disease Control, Dalian Ocean University, 52. Heishijiao Street, Shahekou District, Dalian 116023, China. E-mail: qingsongxu2003@163.com; Fax: +86411 84763004; Tel: +8641184763004 ${ }^{b}$ Institute of Animal Nutrition, Sichuan Agricultural University, Chengdu 611130, China. E-mail: hejun8067@163.com; Fax: +86-28-86290920; Tel: +86-13419354223 'Institute of Process Engineering, Chinese Academy of Sciences, Beijing 100190, China ${ }^{d}$ Zhongke Runxin (Suzhou) Biological Technology Co., Ltd., Suzhou 215000, China
}

many advantages such as more accurate quantization, a wider testing range, higher repeatability, and more reliable analysis. ${ }^{7}$ Currently, in order to investigate the novel transcript units and differentially expressed genes (DEGs), the RNA-Seq has been widely applied to domestic animals, such as pig, ${ }^{8}$ cow, ${ }^{9}$ goat, ${ }^{10}$ sheep ${ }^{11}$ and others. In addition, the efficacy of RNA-Seq has also been used in mammalian reproductive tissues, including pig ovary, ${ }^{12}$ pig placenta, ${ }^{13}$ bovine blastocyst, ${ }^{14}$ goat ovary ${ }^{10}$ and sheep ovary. ${ }^{11}$ Therefore, using RNA-Seq technology to study the relationship between nutrition improvement and sow prolificacy trait is the Frontier research of animal nutrition.

Chitosan oligosaccharide (COS), which is a depolymerized product of chitosan, possesses significant applications in the pharmaceutical, food, agricultural and environmental industries. ${ }^{\mathbf{1 5 , 1 6}}$ Particularly, COS has potential applications as a dietary supplement or nutraceutical for animals. ${ }^{17}$ Several studies have demonstrated that dietary supplementation with COS could improve piglets growth performance and intestinal development ${ }^{18}$ while enhancing animal health. ${ }^{19,20}$ Our previous studies also indicated that dietary COS supplementation improved foetal survival and reproductive performance in multiparous sows, ${ }^{21,22}$ and the foetal survival rate in sows after 35 days COS supplementation was elevated by approximately $13.0 \% .^{22}$ Appreciation of the important role of COS in regulating mammalian foetal survival and growth rates has grown steadily in recent years. Maternal COS supplementation provides an important breakthrough for developing strategies to reduce prenatal loss. Nevertheless, understanding the variation in the 
expression of genes responsible for COS-induced foetal survival and growth alterations is in its primitive stage. In this study, we detected the differential expression profiling of the mRNAs in two groups (control sow ovary and COS sow ovary) using RNASeq technology. This work advanced our understanding of the molecular mechanism of COS-induced fecundity, and provided basic data for future studies.

\section{Materials and methods}

\section{Preparation and composition of COS}

COS was prepared by the enzymatic hydrolysis of chitosan according to the method we reported previously. ${ }^{23}$ The products of the enzymolysis were a mixture of several oligosaccharides with a degree of deacetylation over $95 \%$ and an average molecular weight $\leq 1000 \mathrm{Da}$. The percentage composition of COS was $3.7 \%, 16.1 \%, 28.8 \%, 37.2 \%$ and $14.2 \%$, with a degree of polymerization (DP) of 2-6.

\section{Animals treatment and ovary collection}

Twenty-four multiparous sows (Yorkshire; high-prolificacy gilts introduced to China from Canada), whose parities were in the range 3-4, were selected from a commercial pig farm (Leshan, China) and transported to Sichuan Agricultural University (Chengdu, China). The sows were individually housed in gestation crates $(1.5 \times 2.0 \mathrm{~m})$ in a pregnancy room. The ambient temperature in the pregnancy room was maintained at 15$18{ }^{\circ} \mathrm{C}$. All sows were determined to be in the oestrous stage and then were inseminated twice with unfrozen semen via artificial insemination 3-5 days after weaning. The sows were randomly allotted to one of two treatments (12 sows/treatment) from day 1 of mating to ensure that each group had the same number of sows of similar parity. The treatment groups were as follows: (1) control diet without supplementation (CON); (2) control diet with $\mathrm{COS}$ added at a concentration of $100 \mathrm{mg} \mathrm{kg}^{-1}$ (COS). The sows were fed twice daily either $2.2 \mathrm{~kg}$ of control or COSsupplemented diets during days 1 to 34 of gestation (at $08: 00$ and $18: 00$ ). In addition, all sows were given ad libitum access to water.

At day 35 of gestation, 12 hours after their last meal, six sows were euthanized with an intravenous injection of pentobarbital sodium (50 mg kg-1 body weight) for $15 \mathrm{~min}$ and then the abdomens were immediately opened. Their intact ovaries were rapidly harvested from their carcasses and immediately frozen in liquid nitrogen. All tissue samples were stored at $-80^{\circ} \mathrm{C}$ until the total RNA extraction procedure was performed.

\section{Library preparation and sequencing}

The ovaries were completely ground and total RNA was extracted using TRIzol (Invitrogen, Carlsbad, CA, USA). The quality of the total RNA (RNA Integrity Number $\geq 7$ ) was checked using the Agilent 2100 Bioanalyzer system (Santa Clara, CA, USA). Total RNA from three ovary samples was pooled prior to library preparation in the two experimental groups. Equimolar quantities of RNA from each ovary sample were combined into one pool. According to the manufacturer's manual, sequencing libraries were performed at Beijing Genomics Institute (BGI, Shenzhen, China) using the Illumina Truseq RNA Sample Preparation Kit (Illumina, San Diego, USA). Briefly, mRNA was first extracted from total RNA using oligo (dT) magnetic beads and sheared into short fragments of about 200 bases. These fragmented mRNAs were then used as templates for cDNA synthesis. The cDNAs were then PCR amplified to complete the library. The cDNA libraries were sequenced using an Illumina HiSeq $^{\mathrm{TM}} 2000$ platform.

\section{Bioinformatics analysis}

All clean reads were obtained by rejecting low quality sequence or sequencing adapters, and reads with more than $10 \%$ unknown nucleotides $(\mathrm{N})$. The clean reads were aligned to the gene sequences (downloaded from NCBI database) and pig genome (Sus scrofa 10.2) through TopHat software, ${ }^{24}$ allowing up to two base mismatches. Unmapped or multi-position matched reads were excluded from further analyses. Moreover, sequence saturation analyses of the two libraries were executed to provide an overview of the project.

\section{Expression profiling}

The number of mapped reads for each gene was normalized and calculated by using the reads per kilo bases per million reads (RPKM) method, which is an effective method for eliminating the influence of sequencing discrepancy and gene length. ${ }^{25}$ The false discovery rate (FDR) $\leq 0.001$ and $\mid \log _{2}$ ratio $\mid \geq 1$ were used to identify differentially expressed genes (DEGs). ${ }^{26}$

\section{Gene ontology and pathway enrichment analysis of differentially expressed genes}

All DEGs were submitted to the databases of Gene Ontology (GO) and Kyoto Encyclopedia of Genes and Genomes (KEGG) for enrichment analysis. GO analysis was performed using the Blast $2 \mathrm{GO}$ software $^{27}$ to annotate the function of these DEGs. In addition, the KEGG database was used for the DEGs enriched pathway analysis (http://www.genome.ad.jp/kegg/). Pathways with a $Q$ value $\leq 0.05$ was defined as a significantly enriched pathway in terms of DEGs.

\section{Validation of RNA-Seq data}

To validate the DEGs identified by RNA-Seq, nine genes (Table 2) revealed to be differentially expressed were chosen randomly for qPCR validation. Ovarian total RNA isolation, cDNA synthesis and qPCR analysis were conducted as previously described. ${ }^{28}$ The primers used for qPCR analysis were listed in Table 2 with $\beta$-actin identified as a reference control. The qPCR reactions were performed on an ABI StepOnePlus ${ }^{\mathrm{TM}}$ Real-Time PCR System (ABI, USA) using a SYBR Green qPCR Mix (Takara, Dalian, China) in accordance with the manufacturer's instructions. The thermal cycling conditions were $95{ }^{\circ} \mathrm{C}$ for $10 \mathrm{~min}$, followed by 40 cycles of $95{ }^{\circ} \mathrm{C}$ for $15 \mathrm{~s}$ and $60{ }^{\circ} \mathrm{C}$ for $1 \mathrm{~min}$. Relative quantification analyses were used the comparative CT method, and relative gene expression levels were calculated using the $2^{-\Delta \Delta C_{\mathrm{T}}}$ method. ${ }^{29}$ 


\section{A Composition of Raw Reads (Control)}

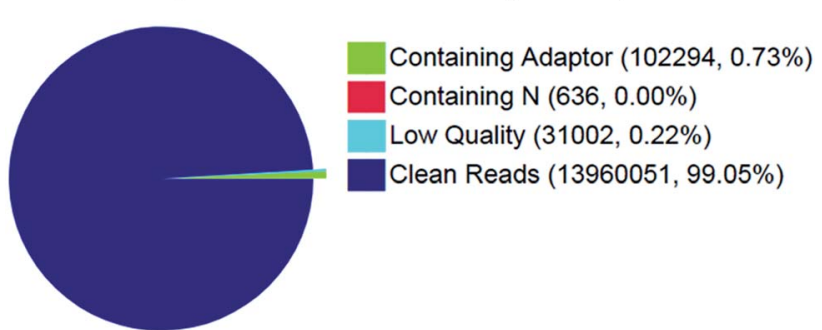

B Composition of Raw Reads (COS)

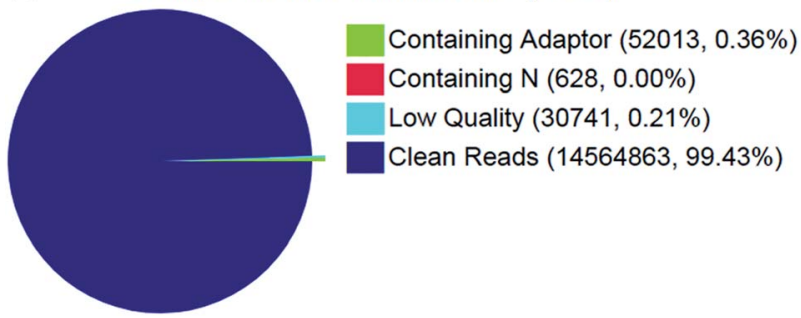

Fig. 1 Composition of total raw reads from the control sow ovary (A) and COS sow ovary (B) libraries.

\section{Results}

\section{Overview of sequencing data}

In the present study, we constructed two RNA-Seq libraries from the control sow ovary and COS sow ovary, respectively. After filtering the adaptor sequences, low quality sequences and regions containing $\mathrm{N}$ sequences, the two libraries still generated over $1.39 \mathrm{~Gb}$ clean reads in each library, and the percentages of clean reads among the raw reads reached $99.05 \%$ and $99.43 \%$ in the control ovary and COS ovary libraries, respectively (Fig. 1), which demonstrated that the two libraries were of high-quality. The major characteristics of the two libraries were analyzed in Table 1 . The result showed varying amount of sequencing reads for these samples. In both libraries, although $59.30 \%$ of the reads in control sow and $56.92 \%$ of the reads in COS sow could be mapped to reference genes, approximately $74 \%$ of the reads mapped to pig genome (control sow ovary with $73.50 \%$, COS sow ovary with $74.57 \%$ ). For the unique match, a little more than $45 \%$ and $66 \%$ of the reads corresponded to reference genes and genome respectively. Moreover, $48.45 \%$ of the reads in control sow and $46.70 \%$ of the reads in COS sow could be perfectly matched to the reference genes, and approximately $58 \%$ of the reads perfectly matched to genome. In addition, the results of saturation analyses (Fig. 2) demonstrated that when the number of sequenced reads reached $2.5 \mathrm{M}$ or more, the number of detected genes almost ceased increasing, which validated the integrity of the libraries for use in further analysis.

\section{Identification and analysis of DEGs}

The RPKM method was adopted to evaluate the gene expression levels. As a result, 17607 and 18014 reference genes were identified from control sow ovary and COS sow ovary libraries, respectively, which shared 16741 genes in common. As shown in Fig. 3, 25\% of the reference genes had 90-100\% coverage, and $12 \%$ of the genes had $80-90 \%$ coverage in control ovary and COS ovary libraries, suggesting that the read distributions were similar between the two libraries. To identify the significance of differences in expressed genes, FDR $\leq 0.001$ and the absolute value of $\log _{2}$ ratio $\geq 1$ were defined as the threshold. A total of 486 significantly differentially expressed genes were identified between the two libraries, with 234 genes up-regulated and 252 genes down-regulated in COS ovary compared with control ovary (Fig. 4).

\section{Gene ontology and pathway enrichment analysis}

The enrichment of DEGs in GO terms was analyzed to obtain insights into the biological implications. Several GO terms significantly enriched for DEGs were investigated and shown in Fig. 5. The GO annotation demonstrated that the DEGs were involved in many biological processes, such as cellular process, single-organism process, metabolic process, biological regulation, response to stimulus, multicellular organismal process, signaling, and developmental process. The main functional groups of DEGs in cellular component were cell, cell part, organelle, membrane, and organelle part, and in molecular function were binding and catalytic activity.

According to the KEGG pathway database, the pathway analysis was performed to predict the significantly enriched signal transduction pathways and metabolic pathways in DEGs. After pathway enrichment analysis, 409 DEGs had been annotated in KEGG pathway. The results indicated that the significant signaling pathways were 34 pathways. For example cell cycle, progesterone-mediated oocyte maturation, p53 signaling

Table 1 A summary of the sequencing reads alignment to the Sus scrofa genome and reference genes

\begin{tabular}{|c|c|c|c|c|}
\hline \multirow[b]{2}{*}{ Sample } & \multicolumn{2}{|l|}{ Alignment to genome } & \multicolumn{2}{|c|}{ Alignment to reference genes } \\
\hline & Control & COS & Control & COS \\
\hline Total reads & 13960051 & 14564863 & 13960051 & 14564863 \\
\hline Total base pairs & 684042499 & 713678287 & 684042499 & 713678287 \\
\hline Total mapped reads & $10261061(73.50 \%)$ & $10860921(74.57 \%)$ & $8278882(59.30 \%)$ & $8289979(56.92 \%)$ \\
\hline Perfect match & $8048824(57.66 \%)$ & $8581743(58.92 \%)$ & $6763308(48.45 \%)$ & $6802338(46.70 \%)$ \\
\hline$\leq 2 \mathrm{bp}$ Mismatch & $2212237(15.85 \%)$ & $2279178(15.65 \%)$ & $1515574(10.86 \%)$ & $1487641(10.21 \%)$ \\
\hline Unique match & $9332910(66.85 \%)$ & $9875898(67.81 \%)$ & $6605864(47.32 \%)$ & $6605721(45.35 \%)$ \\
\hline Multi-position match & $928151(6.65 \%)$ & $985023(6.76 \%)$ & $1673018(11.98 \%)$ & $1684258(11.56 \%)$ \\
\hline Total unmapped reads & $3698990(26.50 \%)$ & $3703942(25.43 \%)$ & $5681169(40.70 \%)$ & $6274884(43.08 \%)$ \\
\hline
\end{tabular}



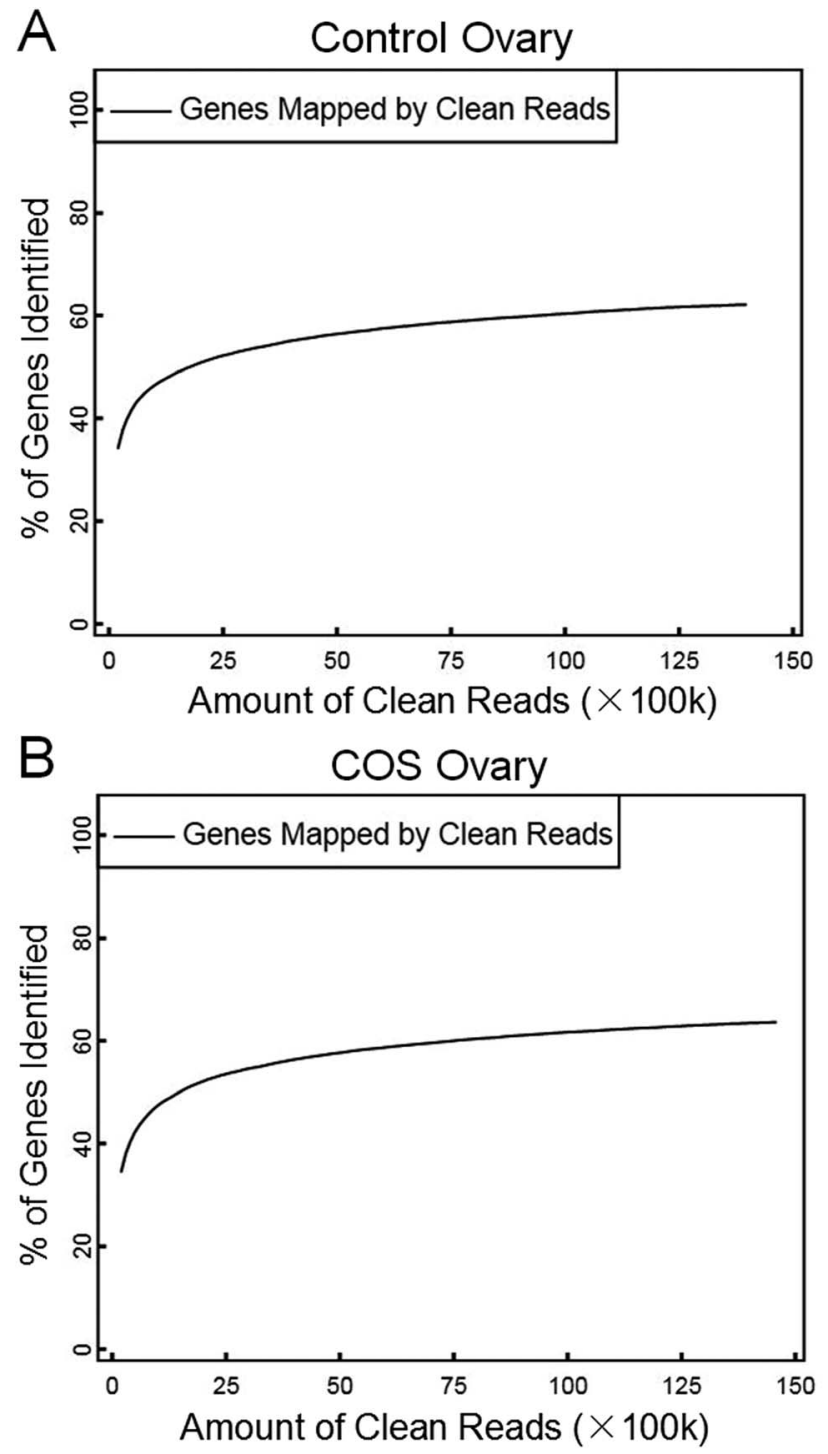

Fig. 2 Saturation description of control sow ovary (A) and COS sow ovary (B). The number of detected genes continued increasing as the total number of sequencing reads increased. When the number of reads reached a certain amount, the number of detected genes almost ceased increasing.

pathway, DNA replication, metabolic pathways, oocyte meiosis, and hematopoietic cell lineage were among the most enriched pathways (Fig. 6).

\section{Confirmation of DEGs by real-time quantitative PCR}

Nine DEGs were randomly selected for qPCR analysis to validate the expression patterns obtained by RNA-Seq. The results indicated that relaxin 2 (RLN2), lysozyme (LYZ), wnt family member 2 (WNT2), integrin subunit beta like 1 (ITGBL1), and endothelin receptor type $\mathrm{B}$ (EDNRB) were up-regulated and surfactant protein C (SFTPC), matrix metallopeptidase 9 (MMP9), E2F transcription factor 1 (E2F1), and cyclin B1 (CCNB1) were down-regulated in COS ovary samples (Table 2), which were basically consistent with the RNA-Seq results.
A Distribution of Genes' Coverage (Control)
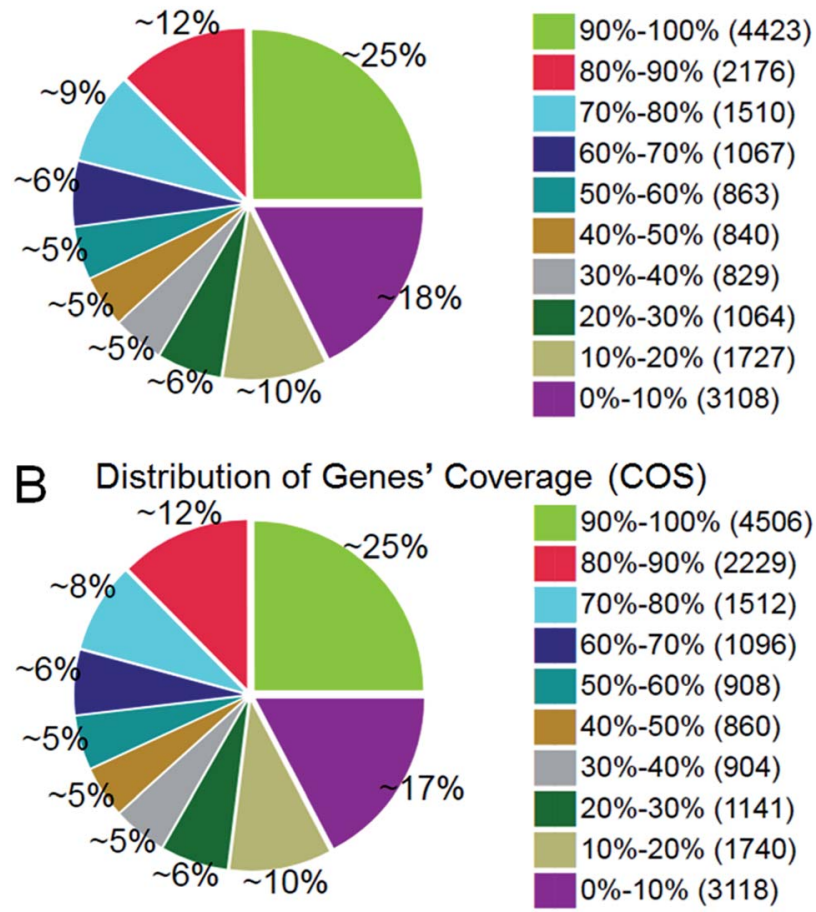

Fig. 3 Distribution of genes coverage in the two sow ovary libraries. Gene coverage was the percentage of a gene covered by reads. This value was equal to the ratio of total base count in a gene covered by uniquely mapped reads to the total base count for that gene.

Therefore, RNA-Seq can be used to reliably and accurately perform for mRNA differential expression analysis.

\section{Discussion}

As the safe and burgeoning feed additive, COS has attracted more and more attentions recently, because it is not only easily soluble in water (generally, the MW of COS is $10 \mathrm{kDa}$ or less) and

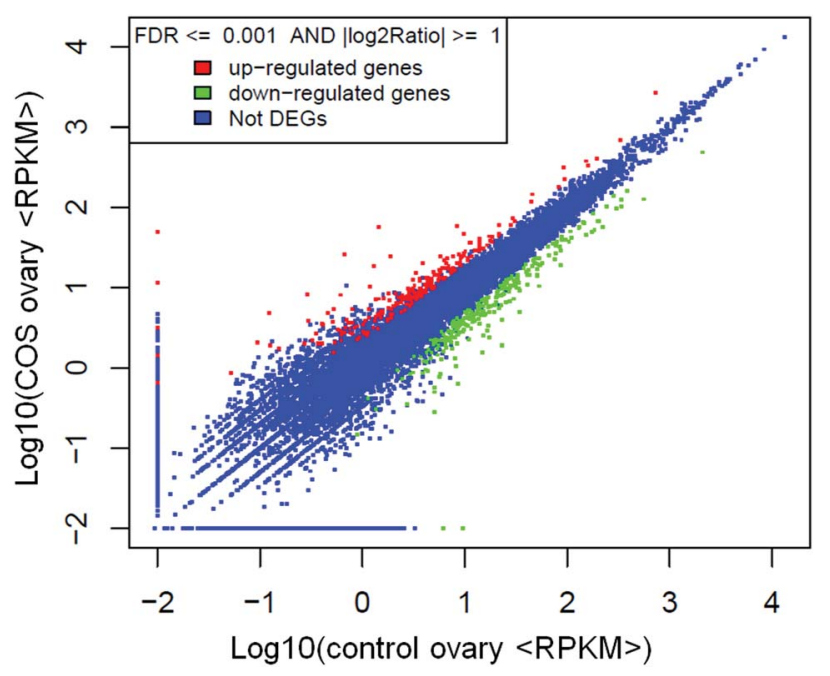

Fig. 4 Scatter plot indicated the comparative results of log transformed gene expression levels and differentially expressed gene distributions between the two libraries. 


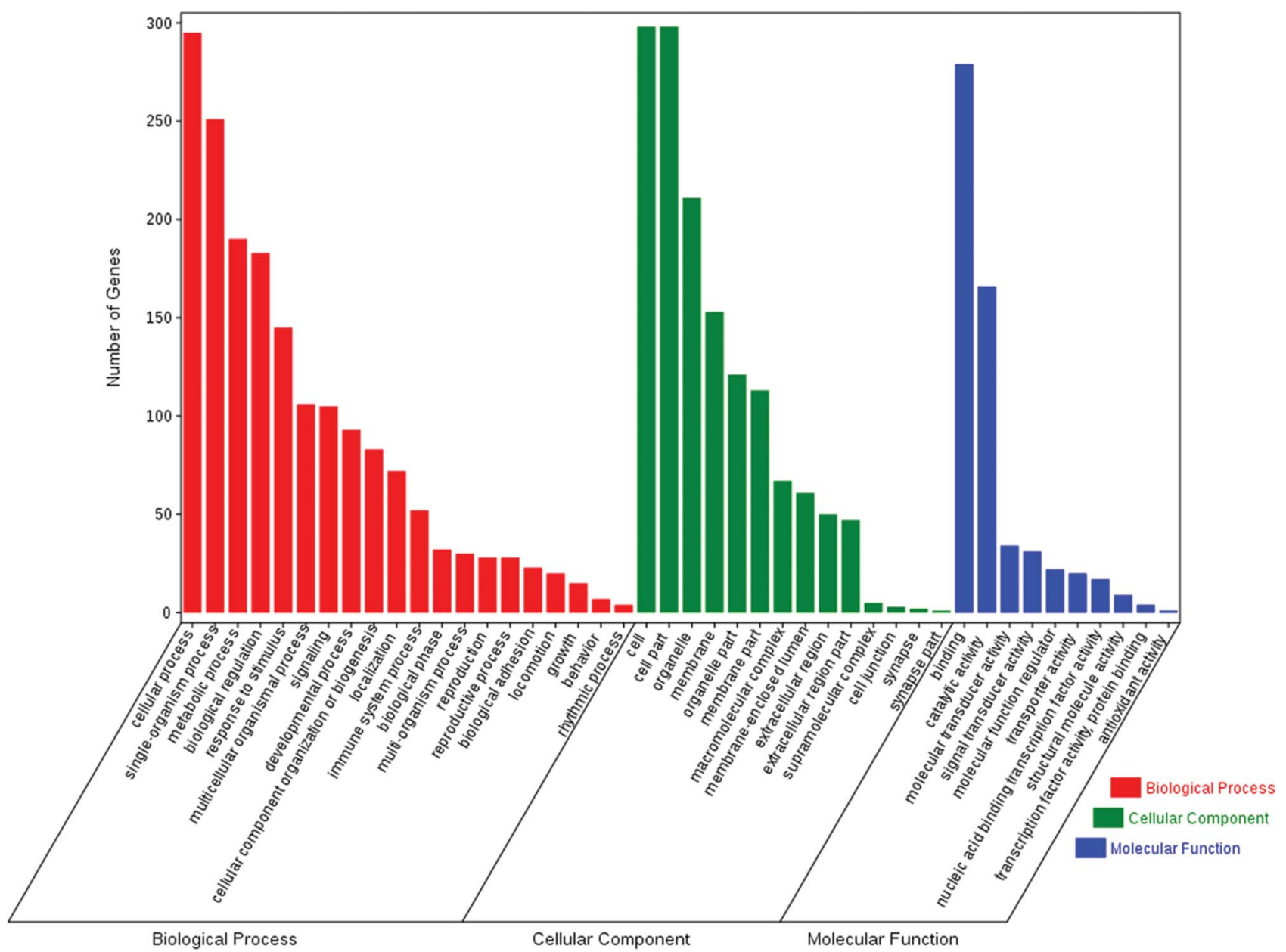

Fig. 5 GO analysis of DEGs between the control and COS ovary libraries. The DEGs were classified into three categories: cellular component, molecular function, and biological process. The number of genes in each category were shown above.

free amino groups in D-glucosamine units, but also readily absorbed through the intestine, quickly getting into the blood flow. ${ }^{30}$ Most importantly, $\operatorname{COS}$ is renewable, non-toxic, biocompatible, and biodegradable. ${ }^{15}$ Our previous study demonstrated that COS supplementation increased the total number of piglets born by $18.5 \%$, the number of piglets born alive by $19.2 \%$, and the live born litter weight by $31.3 \%{ }^{21}$ Moreover, we found the foetal survival rate in pregnant sows after 35 days COS supplementation was elevated by approximately $13.0 \% .^{22}$ However, the underlying molecular mechanism of $\mathrm{COS}$ feed additive in pregnant sow remains largely unknown, especially the relative genes variation. As the ovary directly mediates ovulation and affects litter size, it has a significant impact on the fecundity of mammals. ${ }^{31}$ In the present study, we identified 486 differentially expressed genes in ovaries in control sow and COS sow groups using RNA-Seq technology. A large number of these DEGs were involved in the terms cellular process, cell \& cell part and binding. Furthermore, pathway analysis indicated that these DEGs were significantly enriched in cell cycle, progesterone-mediated oocyte maturation, metabolic pathways, oocyte meiosis, or hematopoietic cell lineage and so on.
Previous studies of pig ovaries indicated that the most differentially expressed genes identified by RNA-Seq were likely to be significant for improving litter size. ${ }^{12}$ In this study, 486 DGEs in ovaries of control sow and COS sow groups were identified by RNA-Seq. Some of the DGEs corresponding to genes previously were involved in prolificacy processes, such as relaxin $2{ }^{32}$ placenta specific 8 (PLAC8), ${ }^{33}$ wnt family member 2 $(\mathrm{WNT} 2)^{34}$ and vascular endothelial growth factor (VEGF) ${ }^{35}$ were up-regulated in COS sow ovary. In animals, relaxin softens the cervix (cervical ripening), and relaxes the uterine musculature. Thus, for a long time, relaxin has been regarded as a pregnancy hormone. ${ }^{32}$ PLAC8 has been investigated in embryo development in different species, and its distribution in cells is dynamic and highly regulated in a manner depending on the developmental stage and cell type. ${ }^{33}$ The Wnt gene family consists of structurally related genes that encode secreted signaling proteins involved in the Wnt signaling pathway. These proteins have been associated with several developmental processes, including regulation of cell fate and patterning during embryogenesis. ${ }^{34}$ It has been demonstrated that VEGF system constitute the most important signaling pathway in angiogenesis, and play an important role in the female ovulatory cycle including follicular development, ovulation, and 


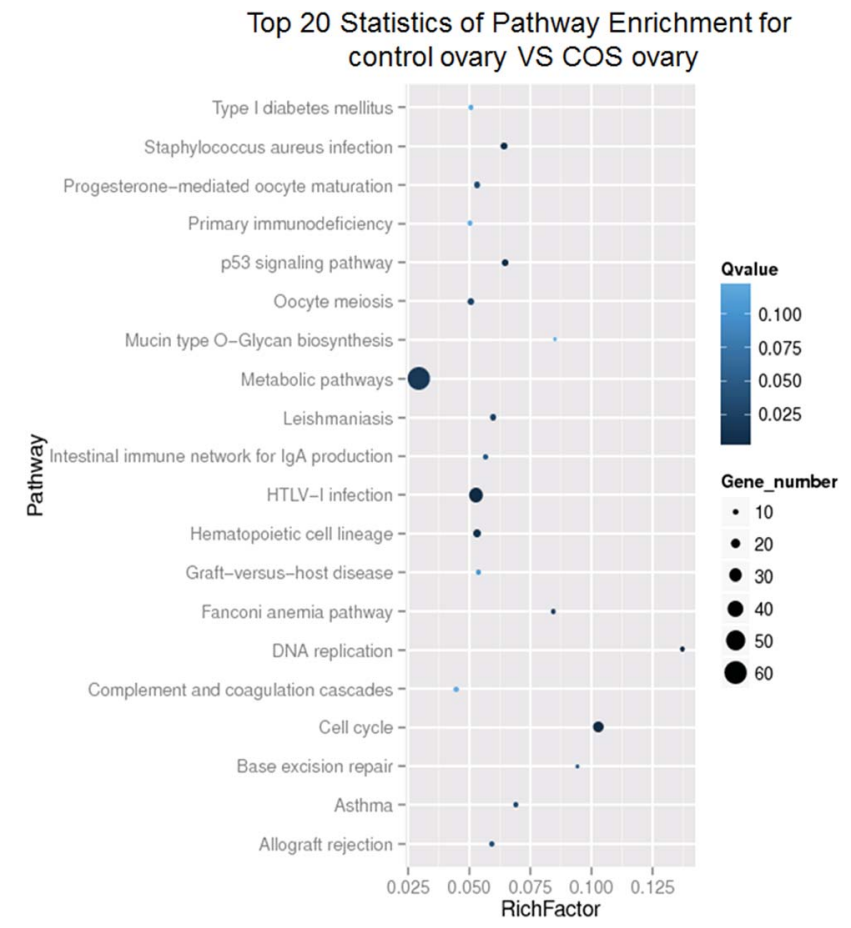

Fig. 6 KEGG enrichment pathway analysis from DEGs. The ordinate represented the enriched pathway terms, and the abscissa represented the richness factor of these terms. Spot size represented the number of differentially expressed genes enriched in each pathway, while the color shade of the spot represented the $Q$ value of each pathway (its less value means greater intensiveness).

corpora lutea formation. ${ }^{35}$ Furthermore, it is now increasingly clear that the feto-placental unit survival and growth are influenced by a complex interactive network of cytokines, some of which are produced by local immune components and others by reproductive tissues. ${ }^{36}$ In contrast to control sow, there was a particularly high overrepresentation of genes related to the immune response in COS sow, such as CD19, CD48, CD84, CD3E, C3a receptor, C5, and lysozyme.

To investigate the biological functions of the DEGs, we performed the GO annotation and KEGG pathway analysis. The results demonstrated that some DEGs between control and COS sows were mainly in the cell cycle, progesterone-mediated oocyte maturation, oocyte meiosis, hematopoietic cell lineage, or metabolic pathways, and so on. Oocyte maturation and early embryo development require precise coordination between cell cycle progression and the developmental programme. The tempo of oocyte meiotic and embryonic mitotic divisions is set by the rate of cyclin B accumulation and the timing of its destruction. ${ }^{37}$ Progesterone production from the corpus luteum is critical for oocyte maturation and natural reproduction. Luteal phase deficiency in natural cycles is an important cause of infertility and pregnancy loss. ${ }^{38}$ It is well established that pubertal activation of the reproductive axis and maintenance of fertility are critically dependent on the magnitude of body energy reserves and the metabolic state of the organism. ${ }^{39}$ As paradigmatic example, much has been learned on the reproductive roles of key metabolic hormones (such as leptin, insulin and ghrelin). ${ }^{40}$ The DEGs were not only related to reproductionrelated pathways but also in those associated with nutrient metabolism. This indicated that the genes might be involved in both reproduction and metabolism. The molecular regulation of animal traits is very complex and the relationships between genes and traits are usually that of "many-to-one" or "one-tomany". ${ }^{12}$ It was readily appreciated that, most physiological processes interacted with each other in the life of organisms.

Table 2 Validation of selected RNA-Seq genes expression by real-time RT-PCR analysis

\begin{tabular}{|c|c|c|c|c|c|}
\hline \multirow[b]{2}{*}{ Gene ID } & \multirow[b]{2}{*}{ Description } & \multicolumn{2}{|c|}{$\begin{array}{l}\log _{2} \text { Ratio (COS ovary/ } \\
\text { Control ovary) }\end{array}$} & \multirow[b]{2}{*}{ Regulation } & \multirow[b]{2}{*}{ Primer sequence } \\
\hline & & RNA-Seq & qPCR & & \\
\hline ENSSSCT00000005749 & Relaxin 2 (RLN2) & 5.30 & 4.02 & Up & $\begin{array}{l}\text { F:CTGAAGGCAACATTGTCTGA } \\
\text { R:TCTCTTTTTTCTGGAATGTTTAT }\end{array}$ \\
\hline ENSSSCT00000000530 & Lysozyme (LYZ) & 3.85 & 3.13 & Up & $\begin{array}{l}\text { F:GCCAAGTGGGAAAGTGA } \\
\text { R:AGGTCATCGTCCAGCAA }\end{array}$ \\
\hline ENSSSCT00000018104 & $\begin{array}{l}\text { Wnt family member } \\
2 \text { (WNT2) }\end{array}$ & 2.09 & 1.50 & Up & $\begin{array}{l}\text { F:TGTGACCCGAAGAAGAAGG } \\
\text { R:ACCGCTTTACAGCCTTCC }\end{array}$ \\
\hline ENSSSCT00000010444 & $\begin{array}{l}\text { Integrin subunit beta } \\
\text { like } 1 \text { (ITGBL1) }\end{array}$ & 1.83 & 1.11 & Up & $\begin{array}{l}\text { F:AGACCTACGACGGCAGCAC } \\
\text { R:TACTTTTTTTCTTGGTCAGGTCAC }\end{array}$ \\
\hline ENSSSCT00000010390 & $\begin{array}{l}\text { Endothelin receptor type } \\
\text { B (EDNRB) }\end{array}$ & 1.37 & 1.42 & Up & $\begin{array}{l}\text { F:TCCGTGCGAAGGACCCA } \\
\text { R:ATGTGAAGCAGGTCTCCCAG }\end{array}$ \\
\hline ENSSSCT00000010544 & $\begin{array}{l}\text { Surfactant protein C } \\
\text { (SFTPC) }\end{array}$ & -3.60 & -2.23 & Down & $\begin{array}{l}\text { F:AGAAACATACTGAGATGGTCCTA } \\
\text { R:AGCCGCTGGTAGTCATAGA }\end{array}$ \\
\hline ENSSSCT00000008139 & $\begin{array}{l}\text { Matrix metallopeptidase } \\
9 \text { (MMP9) }\end{array}$ & -2.52 & -2.29 & Down & $\begin{array}{l}\text { F:AGCCCTGCGTGTTTCCA } \\
\text { R:CGAGTTGCCTCCCGTCA }\end{array}$ \\
\hline ENSSSCT00000007953 & $\begin{array}{l}\text { E2F transcription factor } \\
1 \text { (E2F1) }\end{array}$ & -1.98 & -1.86 & Down & $\begin{array}{l}\text { F:CTGACCACCAAACGCTTCC } \\
\text { R:TGCCTAGCCACTGGATGTG }\end{array}$ \\
\hline ENSSSCT00000024108 & $\begin{array}{l}\text { Cyclin B1 (CCNB1) } \\
\beta \text {-Actin }\end{array}$ & -1.82 & -1.49 & Down & $\begin{array}{l}\text { F:CAAATCAGGCAGATGGAAAT } \\
\text { R:TCTGAGAAGGAGGAAAGTGC } \\
\text { F:CGAGCGCTTCCGGTGTCCAG } \\
\text { R:GTGGTCCCGCCAGACAGCAC }\end{array}$ \\
\hline
\end{tabular}




\section{Conclusion}

In summary, we identified 486 DEGs associated with litter size from COS administrated pig ovaries, and a large number of these DEGs were involved in cell cycle, progesterone-mediated oocyte maturation, metabolic pathways, oocyte meiosis, or hematopoietic cell lineage and so on. These novel findings not only furthered our understanding of the molecular mechanisms underlying the beneficial effects of COS on foetal survival and reproductive performance in pregnant sows, but also provided a theoretical basis for developing functional carbohydrates such as COS as green feed additives for the livestock industry.

\section{Conflicts of interest}

The authors declare that they have no competing interests.

\section{Ethical statement}

All animal procedures and sample collection were conducted according to the Regulations for the Administration of Affairs Concerning Experimental Animals (approved by the State Council of the People's Republic of China on October 31, 1988 and promulgated by Decree No. 2 of the State Science and Technology Commission on November 14, 1988, and revised on March 1st, 2017), and was also approved by the Animal Care and Use Committee of Sichuan Agricultural University (Sichuan, China).

\section{Acknowledgements}

We were grateful to all the laboratory members for their technical advice and helpful discussions. This work was supported by grants from the National Natural Science Foundation of China (31372347), Program for Innovative Talents in Higher Education of Liaoning Province (Q. Xu) and Dalian high level talent innovation support program (2015R020).

\section{References}

1 H. Lin, H. Wang, Y. Wang, C. Liu, C. Wang and J. Guo, Genes, 2015, 6, 1330-1346.

2 P. G. Lawlor and P. B. Lynch, Ir. Vet. J., 2007, 60, 359-366.

3 S. W. Kim, A. C. Weaver, Y. B. Shen and Y. Zhao, J. Anim. Sci. Biotechnol., 2013, 4, 26.

4 E. Apper, C. Meymerit, J. Bodin, F. Respondek and A. Wagner, Journal of Animal Research and Nutrition, 2016, $1,18$.

5 X. Zeng, F. Wang, X. Fan, W. Yang, B. Zhou, P. Li, Y. Yin, G. Wu and J. Wang, J. Nutr., 2008, 138, 1421-1425.

6 F. Finotello and B. Di Camillo, Briefings Funct. Genomics, 2015, 14, 130-142.

7 R. Hrdlickova, M. Toloue and B. Tian, Wiley Interdiscip. Rev.: $R N A, 2017,8$, e1364.
8 M. Schroyen and C. K. Tuggle, Mamm. Genome, 2015, 26, 120.

9 M. McCabe, S. Waters, D. Morris, D. Kenny, D. Lynn and C. Creevey, BMC Genomics, 2012, 13, 193.

10 Y. H. Ling, H. Xiang, Y. S. Li, Y. Liu, Y. H. Zhang, Z. J. Zhang, J. P. Ding and X. R. Zhang, Gene, 2014, 550, 148-153.

11 H. Y. Chen, H. Shen, B. Jia, Y. S. Zhang, X. H. Wang and X. C. Zeng, PLoS One, 2015, 10, e0120170.

12 X. Zhang, L. Huang, T. Wu, Y. Feng, Y. Ding, P. Ye and Z. Yin, PLoS One, 2015, 10, e0139514.

13 S. G. Kwon, J. H. Hwang, D. H. Park, T. W. Kim, D. G. Kang, K. H. Kang, I. S. Kim, H. C. Park, C. S. Na, J. Ha and C. W. Kim, PLoS One, 2016, 11, e0153311.

14 J. L. Chitwood, G. Rincon, G. G. Kaiser, J. F. Medrano and P. J. Ross, BMC Genomics, 2013, 14, 350.

15 C. Muanprasat and V. Chatsudthipong, Pharmacol. Ther., 2017, 170, 80-97.

16 P. Zou, X. Yang, J. Wang, Y. Li, H. Yu, Y. Zhang and G. Liu, Food Chem., 2016, 190, 1174-1181.

17 S. Swiatkiewicz, M. Swiatkiewicz, A. Arczewska-Wlosek and D. Jozefiak, J. Anim. Physiol. Anim. Nutr., 2015, 99, 1-12.

18 J. Wan, F. Jiang, Q. Xu, D. Chen, B. Yu, Z. Huang, X. Mao, J. Yu and J. He, RSC Adv., 2017, 7, 9669-9679.

19 S. Suthongsa, R. Pichyangkura, S. KalandakanondThongsong and B. Thongsong, Livest. Sci., 2017, 198, 37-44. 20 C. Xie, X. Wu, C. Long, Q. Wang, Z. Fan, S. Li and Y. Yin, BMC Vet. Res., 2016, 12, 243.

21 L. Cheng, L. Wang, Q. Xu, L. Huang, D. Zhou, Z. Li, S. Li, Y. Du and H. Yin, Livest. Sci., 2015, 174, 74-81.

22 J. Wan, K. Yang, Q. Xu, D. Chen, B. Yu, Y. Luo and J. He, RSC Adv., 2016, 6, 70715-70722.

23 Y. Li, H. Liu, Q. S. Xu, Y. G. Du and J. Xu, Carbohydr. Polym., 2014, 99, 568-578.

24 D. Kim and S. L. Salzberg, Genome Biol., 2011, 12, R72.

25 A. Mortazavi, B. A. Williams, K. McCue, L. Schaeffer and B. Wold, Nat. Methods, 2008, 5, 621-628.

26 L. Wang, Z. Feng, X. Wang, X. Wang and X. Zhang, Bioinformatics, 2010, 26, 136-138.

27 A. Conesa, S. Gotz, J. M. Garcia-Gomez, J. Terol, M. Talon and M. Robles, Bioinformatics, 2005, 21, 3674-3676.

28 J. Wan, F. Jiang, J. Zhang, Q. Xu, D. Chen, B. Yu, X. Mao, J. Yu, Y. Luo and J. He, Sci. Rep., 2017, 7, 44782.

29 T. D. Schmittgen and K. J. Livak, Nat. Protoc., 2008, 3, 11011108.

30 S. Y. Chae, M. K. Jang and J. W. Nah, J. Controlled Release, 2005, 102, 383-394.

31 Z. Pan, J. Zhang, F. Lin, X. Ma, X. Wang and H. Liu, Mol. Biol. Rep., 2012, 39, 10823-10832.

32 T. Dschietzig, C. Bartsch, M. Greinwald, G. Baumann and K. Stangl, Ann. N. Y. Acad. Sci., 2005, 1041, 256-271.

33 M. Li, D. Liu, L. Wang, W. Wang, A. Wang and Y. Yao, Fertil. Steril., 2016, 106, 781-789.

34 R. Janssen and N. Posnien, Gene Expression Patterns, 2014, 14, 55-61.

35 H. M. Fraser, Reprod. Biol. Endocrinol., 2006, 4, 18.

36 H. Engelhardt, B. A. Croy and G. J. King, Biol. Reprod., 2002, 66, 1875-1880. 
37 Z. Polański, H. Homer and J. Z. Kubiak, Results Probl. Cell Differ., 2012, 55, 69-91.

38 T. B. Mesen and S. L. Young, Obstet. Gynecol. Clin. North Am., 2015, 42, 135-151.
39 J. Roa and M. Tena-Sempere, Mol. Cell. Endocrinol., 2014, 397, 4-14.

40 M. J. Vazquez, A. Romero-Ruiz and M. Tena-Sempere, Metabolism, 2015, 64, 79-91. 